\title{
On asymptotic stability of the Skyrmion
}

\author{
Piotr Bizon, ${ }^{1}$ Tadeusz Chmaj, ${ }^{2,3}$ and Andrzej Rostworowski ${ }^{1}$ \\ ${ }^{1}$ M. Smoluchowski Institute of Physics, Jagiellonian University, Kraków, Poland \\ ${ }^{2}$ H. Niewodniczanski Institute of Nuclear Physics, Polish Academy of Sciences, Kraków, Poland \\ ${ }^{3}$ Cracow University of Technology, Kraków, Poland
}

(Dated: September 28, 2018)

\begin{abstract}
We study the asymptotic behavior of spherically symmetric solutions in the Skyrme model. We show that the relaxation to the degree-one soliton (called the Skyrmion) has a universal form of a superposition of two effects: exponentially damped oscillations (the quasinormal ringing) and a power law decay (the tail). The quasinormal ringing, which dominates the dynamics for intermediate times, is a linear resonance effect. In contrast, the polynomial tail, which becomes uncovered at late times, is shown to be a nonlinear phenomenon.
\end{abstract}

Introduction. Stable stationary solutions are natural candidates for the endstates of evolution of many physical systems. The relaxation to these states is well understood for dissipative systems, described by diffusion equations, however for conservative Hamiltonian systems on unbounded domains the problem is much more difficult because there is no local dissipation of energy and convergence to equilibrium is due to dispersion, that is, radiation of excess energy to infinity [1]. Understanding dissipation by dispersion is important physically because the radiation emitted during the approach to equilibrium encodes information about the attractor - this kind of inverse problem has various applications, for instance in identifying black holes via gravitation radiation emitted during the last stages of gravitational collapse.

In this paper we address the problem of relaxation to equilibrium in a very simple setting of the spherically symmetric Skyrme model [2]. This model, apart from its physical relevance in particle physics, is attractive theoretically because the equilibrium state is completely rigid: it has no moduli and no internal degrees of freedom, which makes the mathematical analysis feasible. Yet, despite the simplicity of the model, the relaxation process exhibits a surprising feature: after a transient oscillatory exponential decay (so called quasinormal ringing) which is a linear resonance effect, there proceeds a power law tail which has a nonlinear origin. Pointing out the failure of the linear perturbation theory in capturing the asymptotic dynamics is the main message of this paper. Below, after introducing the model, we first describe the quasinormal ringing using the linear perturbation theory, then we present the numerical evidence for the asymptotic behavior of solutions, and finally we explain the tail using the nonlinear perturbation theory.

Background. Let $M$ be a spacetime with a metric $\eta_{\mu \nu}$ and $N$ be a complete Riemannian manifold with a metric $g_{A B}$. Consider a map $U: M \rightarrow N$ and denote by $S_{\mu \nu}=g_{A B} \partial_{\mu} U^{A} \partial_{\nu} U^{B}$ the pulled back metric. The (generalized) Skyrme model is defined by the lagrangian

$$
L=-\frac{1}{2} S_{\mu}^{\mu}+\frac{1}{4} \alpha^{2}\left(S_{\mu \nu} S^{\mu \nu}-S_{\mu}^{\mu} S_{\nu}^{\nu}\right),
$$

where $\alpha$ is the coupling constant having the dimension of length. In this paper we consider the original Skyrme model [3] where $M$ is the $3+1$ dimensional Minkowski spacetime with the metric $\eta=-d t^{2}+d r^{2}+r^{2} d \omega^{2}$ and $N$ is the 3 -sphere with the round metric $d s^{2}=$ $d F^{2}+\sin ^{2} F d \Omega^{2}$, where $d \omega^{2}$ and $d \Omega^{2}$ are the standard metrics on the unit 2 -sphere. We restrict our attention to corotational maps for which $F=F(t, r)$ and $\Omega=\omega$. For such maps the Euler-Lagrange equations corresponding to (11) reduce to the single nonlinear wave equation (using the abbreviation $w=r^{2}+2 \alpha^{2} \sin ^{2} F$ )

$$
(w \dot{F})-\left(w F^{\prime}\right)^{\prime}+\sin (2 F)+\alpha^{2} \sin (2 F)\left(\frac{\sin ^{2} F}{r^{2}}+F^{\prime 2}-\dot{F}^{2}\right)=0,
$$

where primes and dots denote derivatives with respect to $r$ and $t$, respectively. We are interested in the long time behavior of solutions of this equation for smooth finite energy initial data. Regularity at the origin is ensured by the boundary condition $F(t, 0)=0$. The total conserved energy associated with solutions of equation (2) can be written as the sum $E=E_{\sigma}+E_{S}$, where

$$
\begin{aligned}
& E_{\sigma}=\frac{1}{2} \int_{0}^{\infty}\left[r^{2}\left(\dot{F}^{2}+{F^{\prime}}^{2}\right)+2 \sin ^{2} F\right] d r \\
& E_{S}=\frac{1}{2} \alpha^{2} \int_{0}^{\infty}\left[\sin ^{2} F\left(\dot{F}^{2}+{F^{\prime}}^{2}\right)+\frac{\sin ^{2} F}{r^{2}}\right] d r .
\end{aligned}
$$

The quadratic part of the energy $E_{\sigma}$, corresponding to the pure sigma model $(\alpha=0)$, has the supercritical scaling $E_{\sigma}[F(x / \lambda)]=\lambda E_{\sigma}[F(x)]$, hence for $\alpha=0$ it may be energetically favorable for solutions to shrink and consequently singularities are expected to develop for some initial data [4]. The quartic part of the energy $E_{S}$, introduced by Skyrme, has the subcritical scaling $E_{S}[F(x / \lambda)]=\lambda^{-1} E_{S}[F(x)]$, thus for nonzero $\alpha$ shrinking of solutions to zero size is prevented by energy conservation. Hereafter, we assume that the coupling constant $\alpha$ is nonzero and use the unit of length such that $\alpha=1$. Note that for the total energy to be finite, solutions must satisfy the boundary condition at spatial infinity $F(t, \infty)=m \pi(m=0,1, \ldots)$, where an integer 
$m$ is the topological degree of the map. Since the time evolution is continuous (as long as no singularity forms), this condition breaks the initial value problem into infinitely many disjoint topological sectors labeled by the degree $m$. It is well known that for each $m$ there is a unique regular static solution of equation (2) [5]. Our numerical studies indicate that these static solutions play the role of global attractors in the evolution of regular corotational initial data of a given degree, that is every solution starting from smooth finite energy initial data of degree $m$ remains globally regular for all times and asymptotically settles down to the static solution of degree $m$. Below, for concreteness, we focus our attention on the degree-one sector $m=1$. In this case we shall refer to the static solution as the Skyrmion and denote it by $S(r)$. The Skyrmion is the most interesting corotational soliton because, in contrast to solutions with $m>1$, it is stable with respect to general (nonradial) perturbations. The profile function $S(r)$ (see Fig. 1) is not known explicitly. The existence of the Skyrmion was proved rigorously both by ODE techniques [5] and by variational methods [6].

Linear stability and quasinormal modes. In order to interpret the numerical results shown below we first need to discuss the spectrum the linear perturbations around the Skyrmion. To this end we seek solutions in the form $F(t, r)=S(r)+\delta F(t, r)$. Plugging this into equation (2), linearizing and using the auxiliary field $v(t, r)$ defined by

$$
\delta F(t, r)=\frac{v(t, r)}{\sqrt{r^{2}+2 \sin ^{2} S}},
$$

we get the linear wave equation for the perturbation

$$
\ddot{v}-v^{\prime \prime}+\left(\frac{2}{r^{2}}+V\right) v=0,
$$

where the effective potential is

$$
V=-4 a^{2} \frac{1+3 a^{2}+3 a^{4}}{\left(1+2 a^{2}\right)^{2}}, \quad a=\frac{\sin S}{r} .
$$

Near the origin $S(r) \sim b r$ (with $b \approx 2.0075$ ) and near spatial infinity $S(r) \sim \pi-c / r^{2}$ (with $c \approx 2.1596$ ), hence the potential $V(r)$ is finite at $r=0$ and falls off as $r^{-6}$ for large $r$. Substituting $v(t, r)=e^{-i k t} \psi(r)$ into equation (6) we obtain the $l=1$ radial Schrödinger equation

$$
-\psi^{\prime \prime}+\left(\frac{2}{r^{2}}+V(r)\right) \psi=k^{2} \psi
$$

It is known that this equation has no bound states (and the spectrum is purely continuous $k^{2} \geq 0$ ) which implies that the Skyrmion is linearly stable [7].

Although linear stability is an important property of a soliton, it provides little information about the asymptotic behavior of solutions near the soliton. The key concept in the studies of the asymptotic stability of the soliton is the notion of the quasinormal mode. The quasinormal mode (known also as the resonance) is a regular solution of equation (8) which satisfies the outgoing wave condition for $r \rightarrow \infty$

$$
\psi(r) \sim e^{i k r}, \quad k=\Omega-i \Gamma, \quad \Gamma>0 .
$$

The quasinormal mode with the least damping factor $\Gamma$ is expected to dominate an intermediate stage of the relaxation to the soliton. In order to find this mode we use a shooting method which goes as follows. First, we express $\psi$ in the amplitude-phase form as $\psi=A \exp (i \phi)$ and rewrite equation (8) as the following system

$$
\begin{gathered}
-A^{\prime \prime}+A \phi^{\prime 2}+\left(\frac{2}{r^{2}}+V+\Gamma^{2}-\Omega^{2}\right) A=0, \\
A \phi^{\prime \prime}+2 A^{\prime} \phi^{\prime}-2 \Omega \Gamma A=0 .
\end{gathered}
$$

To ensure regularity at the center we require that

$$
A(r) \sim r^{2} \quad \text { and } \quad \phi(r) \sim \frac{\Omega \Gamma}{5} r^{2} \quad \text { for } \quad r \rightarrow 0 .
$$

We need to find $\Omega$ and $\Gamma$ such that the condition (9) is satisfied. A naive shooting method does not work because an unwanted ingoing wave contamination of the condition (9) decreases exponentially with $r$ and cannot be tracked numerically. To overcome this difficulty (which is intrinsic to the problem and any numerical method has to cope with it) we first solve equations (10a) and (10b) with the initial condition (11) up to some relatively small intermediate $r_{0}$. Next, for $r>r_{0}$ we define the logarithmic derivative $g=\psi^{\prime} / \psi=A^{\prime} / A+i \phi^{\prime}$ and replace the Schrödinger equation (8) by the Ricatti equation

$$
g^{\prime}+g^{2}-\frac{2}{r^{2}}-V+k^{2}=0 .
$$

We solve this equation backwards in $r$ from some large $R$ to $r_{0}$ starting with the initial value

$$
g(R)=\frac{k \hat{h}_{1}^{\prime}(k R)}{\hat{h}_{1}(k R)}
$$

where the Ricatti-Hankel function $\hat{h}_{1}(k r)=\left(-i+\frac{1}{k r}\right) e^{i k r}$ is the exact outgoing wave solution of the free $(V=0) \mathrm{Ri}$ catti equation (12). The value of $R$ should be chosen sufficiently large so that the influence of the potential $V(R)$ is negligible, however in practice $R$ should not be too large in order to avoid numerical instabilities. Matching the logarithmic derivatives at the midpoint $r_{0}$ we found the quasinormal mode at $k=0.61-0.26 i$.

Numerics. We solved equation (2) numerically for different degree-one initial data and found that all solutions remain globally regular and asymptotically settle down to the Skyrmion (see Fig. 1). Proving this asymptotic completeness property is a challenging open problem which we do not pursue here but take it as the starting point for further discussion. 


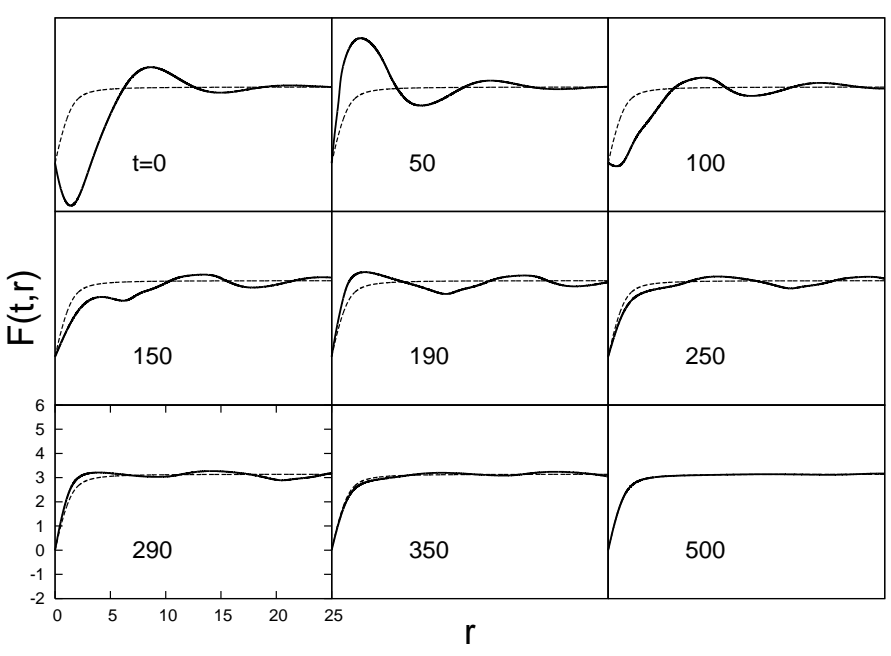

FIG. 1: A solution of degree one (solid line) is shown to converge to the Skyrmion (dashed line).

Our aim is to understand the asymptotic dynamics of convergence to the Skyrmion. Here we mean convergence in the pointwise sense, that is we consider the behavior of a solution $F(t, r)$ for large $t$ at a fixed distance $r$. The basic mechanism of decay is, of course, dispersion - it is clearly seen in Fig. 1 how the excess energy is being radiated away to infinity as the solution approaches the Skyrmion. During the relaxation process one can distinguish two universal stages of evolution: the oscillatory exponential decay which we shall refer to as the quasinormal ringing and the polynomial decay which we shall refer to as the tail. The quasinormal ringing is a wellknown effect in the linear scattering theory [8, 9]. As the name indicates, it is due to the presence of a quasinormal mode. The numerical confirmation of this fact is given in Fig. 2 where we show that for intermediate times the deviation of the solution from the Skyrmion is perfectly approximated by the fundamental quasinormal mode.

For later times the quasinormal mode becomes negligible and the decay takes the form of a polynomial tail. The numerical computation of the tail (see Fig. 3) gives the power-law decay $F(t, r)-S(r) \sim t^{-5}$. Surprisingly, the rate of decay of the tail is different than that predicted by the linear scattering theory. To see this, recall that according to this theory [10], for compactly supported initial data a solution of the linear radial wave equation with a regular potential $V(r)$,

$$
\ddot{v}-v^{\prime \prime}+\frac{l(l+1)}{r^{2}} v+V v=0,
$$

decays at a fixed $r$ as $v(t, r) \sim t^{-\gamma}$, where $\gamma=2 l+\beta$ and $\beta>3$ is the rate of fall-off of the potential at spatial infinity, i.e. $V(r) \sim r^{-\beta}$ for $r \rightarrow \infty$ (for compactly supported or exponentially localized potentials there are no tails). In the case at hand (equation (6)) we have $l=1$ and $\beta=6$, hence according to the linear theory the tail should have the power index $\gamma=8$ instead of

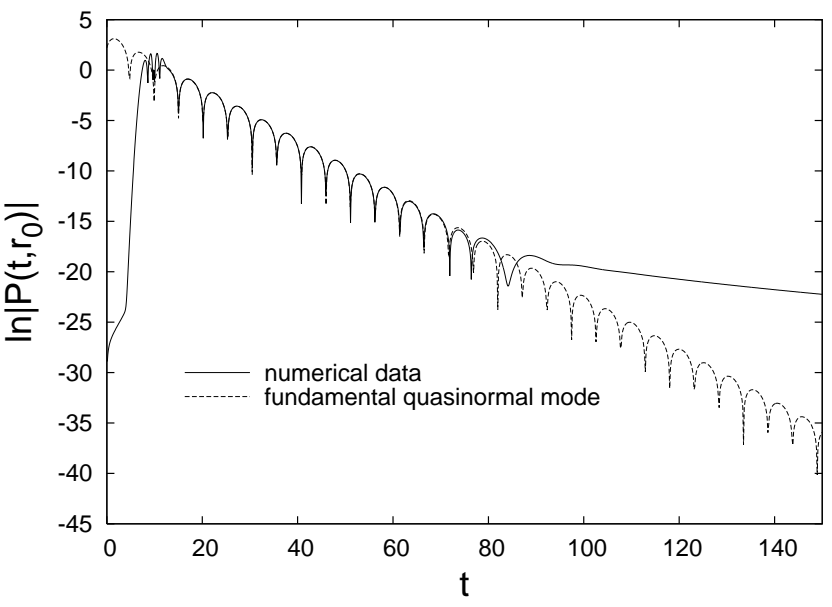

FIG. 2: We plot $\ln \left|P\left(t, r_{0}=10\right)\right|$, where $P(t, r)=w \dot{F}(t, r)$. Fitting the exponentially damped oscillation $P\left(t, r_{0}\right)=$ $A e^{-\Gamma t} \sin (\Omega t+\delta)$ to the numerical data on the time interval $(20,60)$ we get $\Omega=0.610$ and $\Gamma=0.260$ in perfect agreement with the perturbative calculation of the fundamental quasinormal mode.

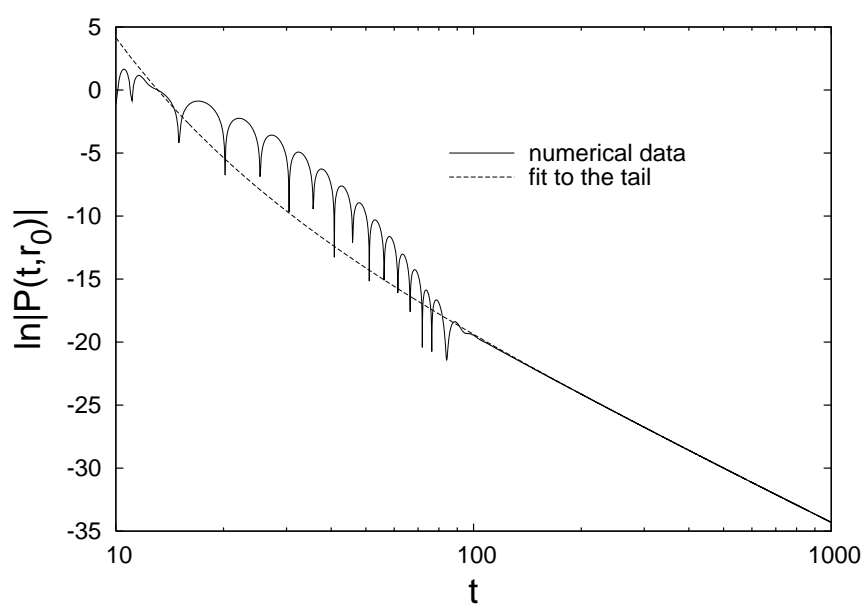

FIG. 3: The same plot as in Fig. 2 but using the log-log scale. Fitting the function $a-b \ln t+c / t$ (the power law decay plus the first correction) to the numerical data on the interval $(200,1000)$ we get $b=6.05$.

the observed $\gamma=5$. Thus, the tail must be a nonlinear effect and to understand it one needs to go beyond the linear perturbation theory. We found that the third order perturbation theory provides a very good approximation of the tail. Unfortunately, the perturbative expansion around the Skyrmion is very messy and the technical details of the calculation might obscure the key mechanism which is responsible for the nonlinear tail. To avoid that, we will take advantage of the fact that the same mechanism is operating in a simpler setting of the relaxation to the vacuum in the topologically trivial sector $m=0$. In this case there is no tail at all at the linear level. 
Nonlinear tail. For topologically trivial initial data all solutions converge asymptotically to the vacuum $F_{0}=0$. To determine the rate of convergence, we substitute the expansion $F=\epsilon F_{1}+\epsilon^{2} F_{2}+\epsilon^{3} F_{3}+\mathcal{O}\left(\epsilon^{4}\right)$ into equation (2), where $\epsilon F_{1}$ satisfies initial data while all $F_{n}$ with $n>1$ have zero data. In the first order we get the free $l=1$ radial wave equation

$$
\mathcal{L} F_{1}=0, \quad \mathcal{L}:=\frac{\partial^{2}}{\partial t^{2}}-\frac{1}{r^{2}} \frac{\partial}{\partial r}\left(r^{2} \frac{\partial}{\partial r}\right)+\frac{2}{r^{2}},
$$

whose general regular solution has the form

$$
F_{1}(t, r)=\frac{a^{\prime}(t-r)+a^{\prime}(t+r)}{r}+\frac{a(t-r)-a(t+r)}{r^{2}},
$$

where the function $a(r)$ is determined by initial data. We assume that the initial data have compact support, hence $F_{1}$ has no tail in agreement with Huygens' principle.

In the second order $\mathcal{L} F_{2}=0$, hence $F_{2}$ vanishes, but in the third order we get the inhomogeneous equation

$$
\begin{gathered}
\mathcal{L} F_{3}=\frac{4}{3 r^{2}} F_{1}^{3}+h, \\
h=\frac{2 \alpha^{2}}{r^{4}}\left(F_{1}^{3}-2 r F_{1}^{2} F_{1}^{\prime}+r^{2} F_{1}\left({F_{1}^{\prime}}^{2}-\dot{F}_{1}^{2}\right)\right) .
\end{gathered}
$$

We solve equation (17) using the retarded Green's function of the operator $\mathcal{L}$

$G\left(t-t^{\prime}, r, r^{\prime}\right)=\left[\left|r-r^{\prime}\right| \leq t-t^{\prime} \leq r+r^{\prime}\right] \frac{r^{2}+r^{\prime 2}-\left(t-t^{\prime}\right)^{2}}{4 r^{2}}$

It follows from (16) that for large $r$ the term $h$ is of lower order in comparison with the first term on the right hand side of equation (17), thus we can drop it without affecting the leading order asymptotics of the tail. Then, using double null coordinates $u=t^{\prime}-r^{\prime}, v=t^{\prime}+r^{\prime}$, we obtain

$$
F_{3}(t, r)=\frac{2}{3 r^{2}} \int_{|t-r|}^{t+r} d v \int_{-v}^{t-r} \frac{(v-t)(t-u)+r^{2}}{(v-u)^{2}} F_{1}^{3}(u, v) d u .
$$

We are interested in the asymptotic behavior of $F_{3}(t, r)$ for a fixed $r$ and $t \rightarrow \infty$ (time-like infinity). Since the initial data have compact support, in this limit we can change the order of integration in (20) and perform the integration over $v$ explicitly. In the leading order we get

$$
F_{3}(t, r) \sim c r t^{-5}, \quad c=-\frac{64}{9} \int_{-\infty}^{\infty} a^{\prime}(u)^{3} d u
$$

where the constant $c$ is the only trace of initial data. We have verified numerically that this formula provides a very good approximation of the tail for solutions having sufficiently small initial data (see Fig. 4).
Conclusions. It should be clear from the above discussion that the nonlinear tail is not an exceptional feature of the Skyrme model but it is a general phenomenon in scattering theory for nonlinear wave equations which will be present whenever the backscattering due to an effective potential around the attractor is weaker that the backscattering due to a nonlinearity. This kind of phenomenon does not seem to have been explored in the literature and we hope that our letter will initiate investigations of an interplay between linear and nonlinear effects in relaxation processes.

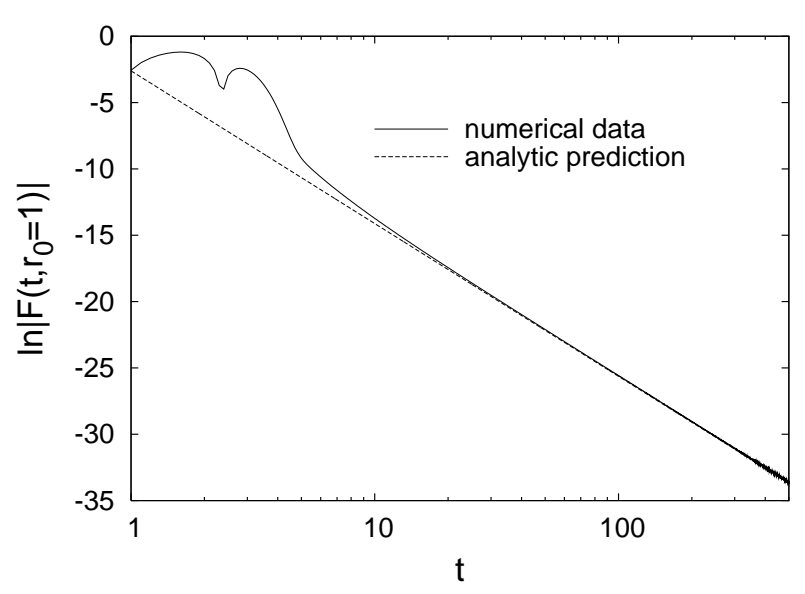

FIG. 4: We plot the degree zero solution (solid line) for initial data $F(0, r)=r^{3} e^{-r^{2}}, \dot{F}(0, r)=0$ and superimpose (dashed line) the analytic prediction for the tail (21) with $c=35 \sqrt{3 \pi} / 1458 \approx 0.0737$ (note that there is no adjustable parameter). The relative error (representing the contribution from higher order iterations) is $\sim 5 \%$.

Acknowledgments: We thank Nikodem Szpak for discussions. This research was supported in part by the Polish Research Committee grant 1PO3B01229.

[1] M.I. Weinstein, Extended Hamiltonian Systems in Handbook of Dynamical Systems, eds. B. Hasselblatt and A. Katok, volume 1B, North Holland (2005).

[2] N. Manton and P. Sutcliffe, Topological Solitons, Cambridge University Press (2004).

[3] T.H.R. Skyrme, Proc. R. Soc. A260, 127 (1961).

[4] P. Bizoń, T. Chmaj, and Z. Tabor, Nonlinearity 13, 1411 (2000).

[5] J.B. McLeod and W.C. Troy, Proc. Roy. Soc. Edinburgh A118, 271 (1991).

[6] L.B. Kapitanski and O.A. Ladyzenskaya, Zap. Nauchn. Semin., LOMI 127, 84 (1983).

[7] M. Heusler, S. Droz, and N. Straumann, Phys. Lett. B271, 61 (1991).

[8] P.D. Lax and R.S. Phillips, Scattering Theory, Academic Press, New York (1967).

[9] K.D. Kokkotas and B.G. Schmidt, Living Rev. Rel. 2, 2 (1999).

[10] E.S.C. Ching et al., Phys. Rev. D52, 2118 (1995). 